\title{
Negative-refraction imaging with two-dimensional phononic crystals
}

\author{
Manzhu Ke, Zhengyou Liu,* Chunyin Qiu, Wengang Wang, and Jing Shi \\ Department of Physics, Wuhan University, Wuhan 430072, People's Republic of China \\ Weijia Wen and Ping Sheng \\ Department of Physics, The Hong Kong University of Science and Technology, Clear Water Bay, Hong Kong
}

(Received 11 May 2005; published 24 August 2005)

\begin{abstract}
We report negative refraction imaging for acoustic waves in a two-dimensional phononic crystal. The sample consists of a triangular array of steel rods immersed in water. Both the observed negative refraction behavior and imaging effect are in excellent agreement with numerical simulations by the multiple scattering theory method. The existence of nearly circular dispersion surfaces for the phonic crystal sample means that the negative refraction is well defined for all angles of incidence, and very robust in its realization and application to focusing and imaging.
\end{abstract}

DOI: 10.1103/PhysRevB.72.064306

PACS number(s): 63.20.-e, 43.35.+d

\section{INTRODUCTION}

The negative refractive index was first proposed as a theoretical possibility in the 1960 's. ${ }^{1}$ A negative refraction index would allow a flat slab of a material to behave as a lens. ${ }^{2}$ There are two types of negative refraction. The first type has simultaneous negative permittivity and negative susceptibility $^{3}$ and the other type arises from the negativedefinite photonic effective mass, ${ }^{4,5}$ in which the photonic crystals have an effective refractive index (ERI) controlled by the band structure.

Recently, the negative refraction behavior and imaging effect in photonic crystals have been experimentally observed. ${ }^{5-11}$ Negative-refraction and imaging effects of a water surface wave by a periodic structure were also theoretically and experimentally demonstrated recently. ${ }^{12}$ The anomalous features of negative refraction open the door to a variety of applications. The interest in photonic crystals motivates the search for analogous phenomena in phononic crystals, ${ }^{13-23}$ which are the elastic and acoustic analogs of photonic crystals. ${ }^{14,15,18}$ In particular, Yang et al. ${ }^{18}$ have recently demonstrated the focusing of ultrasound by a threedimensional phononic crystal, both experimentally and theoretically. In this work, we present a study on the negative refraction and imaging effect by using a two-dimensional (2D) phononic crystal. The advantage of the $2 \mathrm{D}$ system is that it has nearly circular (equifrequency) dispersion surfaces, which makes the negative refraction effect very robust and easily realizable. The measured results are shown to be in excellent agreement with the theory. We have adopted the first-principle multiple scattering theory (MST), ${ }^{24-26}$ both to calculate the phononic crystal band structure in the reciprocal space, and to perform numerical wave simulations as a guide to the experiments. The experimental verification of negative refraction is a step toward the realization of a "superlens" in the acoustic field.

\section{EXPERIMENT AND DISCUSSIONS}

Our phononic crystal consists of a triangular array of steel rods in water. The lattice constant is $a=1.5 \mathrm{~mm}$ and the di- ameter of the steel rods is $d=1 \mathrm{~mm}$. An understanding of negative refraction behavior in a photonic crystal or a phononic crystal can be achieved by looking into the band structure and the equifrequency surface (EFS) of the crystal. ${ }^{17,27-30}$ Figure 1(a) shows the band structure of this 2D phononic crystal along the $\Gamma \mathrm{M}$ direction. The circle dot represents the calculation by the MST method and the solid curves are the experimental result. To obtain the band structure experimentally, we measure the phase delay caused by the sample by directly fast-Fourier transforming the input and transmitted pulses and calculating the phase difference at each frequency. The phase delay is then used to determine
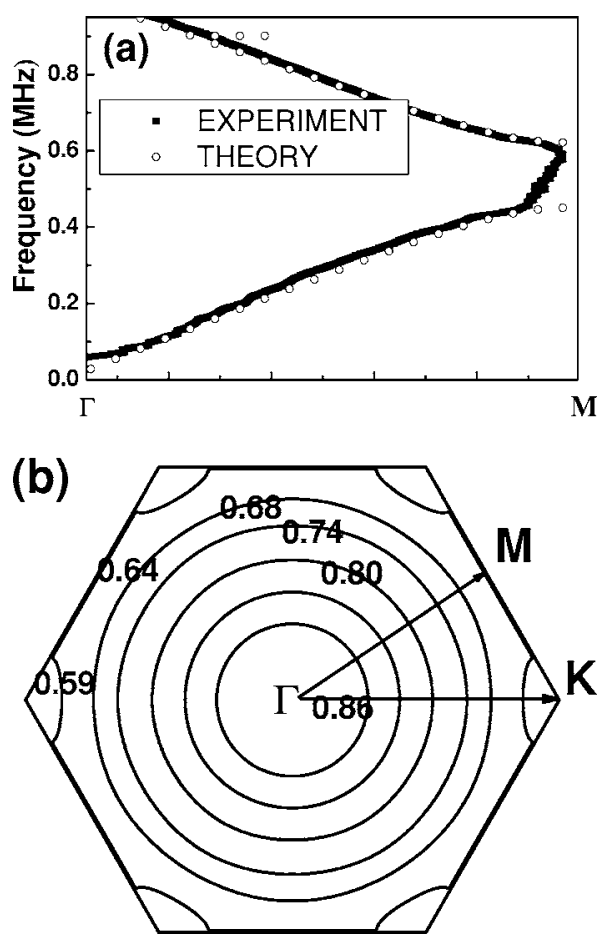

FIG. 1. (a) Band structure for the phononic crystal consisting of a triangular array of steel rods in water; (b) EFS' for frequencies at the second band, moving inward with increasing frequencies. 
(a) Needle hydrophone

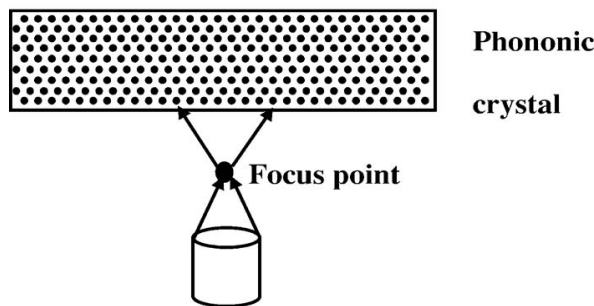

\section{Focus transducer}

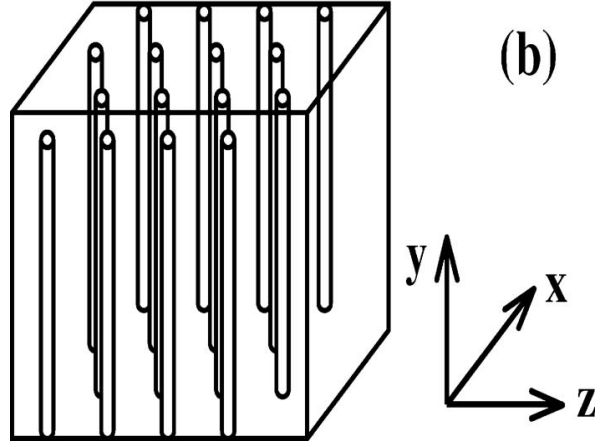

FIG. 2. (a) Experimental setup for the measurements; (b) the rod array in the phononic crystal. the phase velocity of the acoustic wave propagating in the phononic crystal, from which the dispersion relation or the band structure is determined. There is a detailed description for the measurement of the band structure for acoustic waves in phononic crystals in Ref. 31. As can be seen in Fig. 1(a), there is a band gap for frequencies from 0.43 to $0.63 \mathrm{MHz}$. At a given frequency, the resulting intersection of the band structure defines the EFS. Figure 1(b) shows the EFS for some frequencies at the second band. We note that the EFSs have different shapes, but within the frequency range of 0.64-0.95 MHz, the EFSs are nearly circular. From the band structure and EFS of Fig. 1, we can infer that the negativerefraction behavior can occur at the second band for the frequencies from 0.64 to $0.95 \mathrm{MHz}$ because the EFSs move inward with increasing frequencies. ${ }^{3}$ In this case, it is easy to know that $\vec{S} \cdot \vec{k}<0$. Thus, in this frequency range, there exists a well-defined effective refractive index (ERI) for the sonic crystal, which is $n=-|\vec{k}| c / \omega$.

To demonstrate the negative refraction behavior in detail, we measured wave propagation through a nine-layer crystal with a thickness of $11.392 \mathrm{~mm}$. Our experimental setup is based on the well-known ultrasonic transmission technique. Figure 2(a) gives a schematic diagram of the experimental setup, showing the position of the phononic crystal with respect to the generating ultrasonic transducer and the detecting hydrophone. The entire assembly was immersed in water. A pulser/receiver generator (Panametrics model 5900PR) produces a short duration pulse. We used a focus immersion transducer as the generating transducer. The focus transducer was placed at a position such that its focus, which has a size
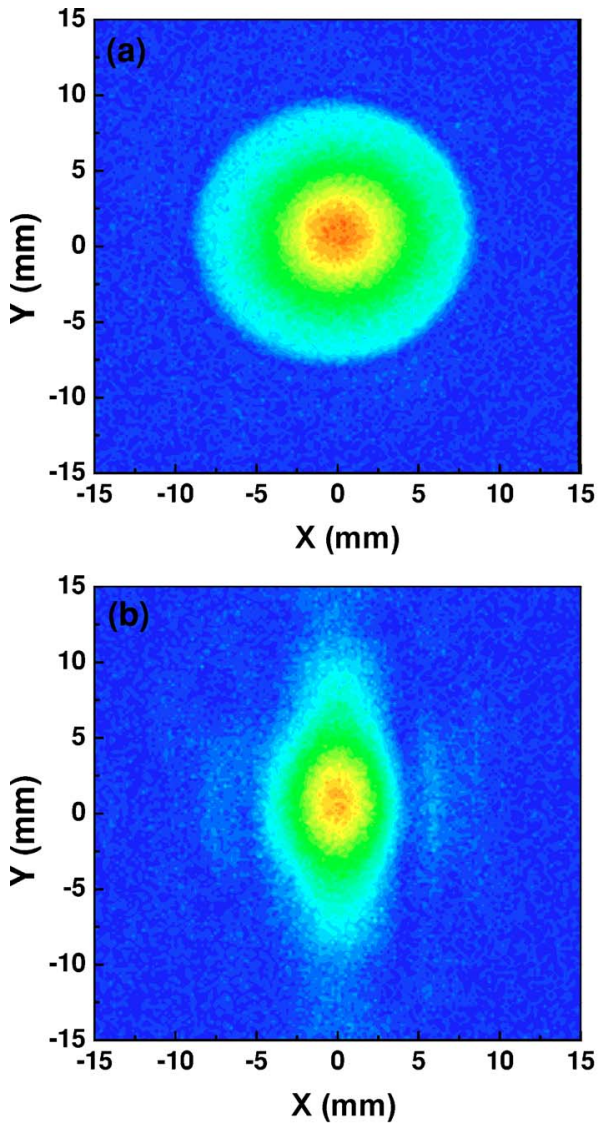
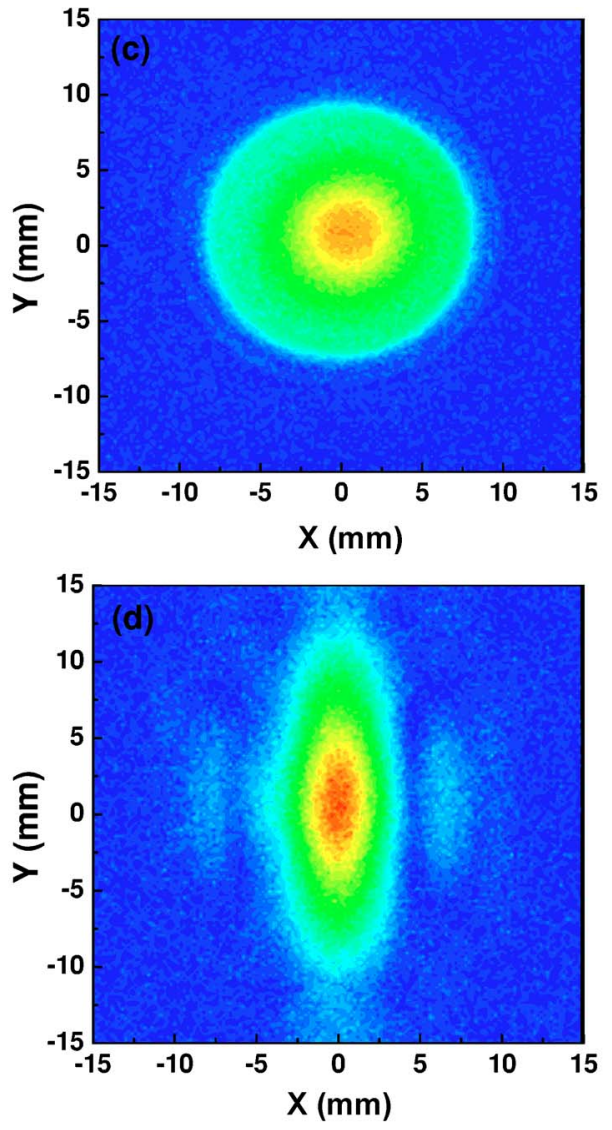

FIG. 3. (Color online) Measured field pattern in a plane parallel to the crystal surfaces (a) without and (b) with a crystal at $20 a$ away from the source for 0.68 $\mathrm{MHz}$; (c) without and (d) with a crystal at $24 a$ away from the source for $0.74 \mathrm{MHz}$. 

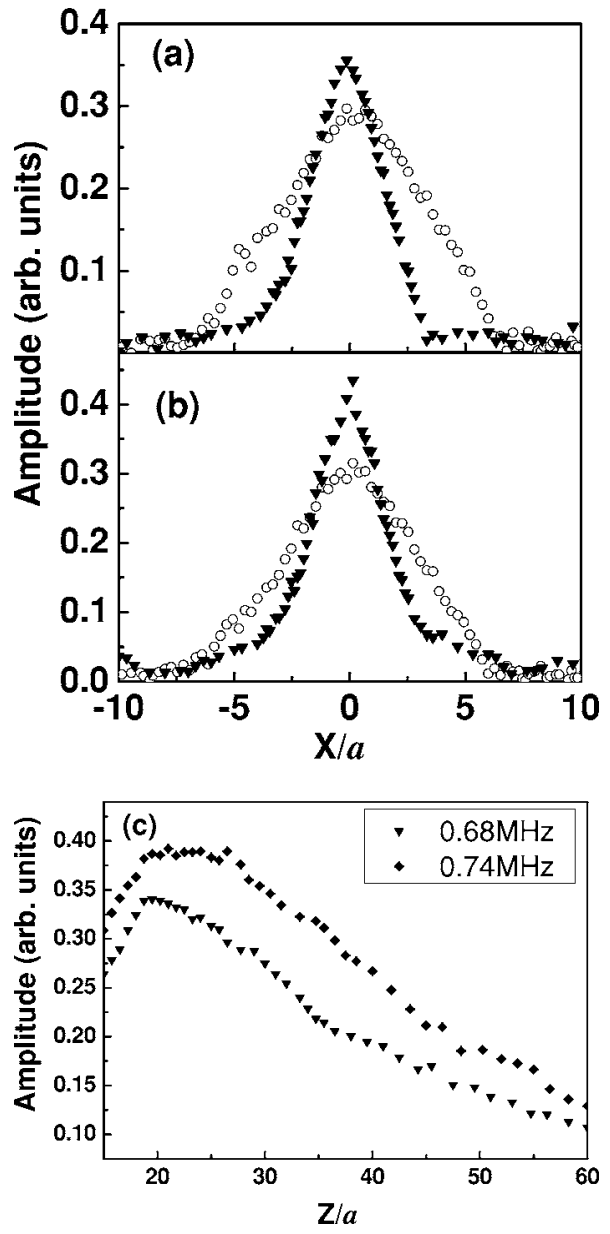

FIG. 4. Pressure distributions (a) at $0.68 \mathrm{MHz}$ along the $X$ direction; (b) at $0.74 \mathrm{MHz}$ along the $X$ direction; (c) at 0.68 and 0.74 $\mathrm{MHz}$ along the $\mathrm{Z}$ direction.

of about $1.5 \mathrm{~mm}$ in diameter and serves as a point source in the experiment, is about five lattice constants from the front surface of the sample. The pulses transmitted through the sample were detected by a polyvinylidence fluoride needle microhydrophone, which had a diameter much less than the ultrasonic wavelength. The pulses were then transformed into the frequency domain using a fast-Fourier-transformed (FFT) technique, allowing the wave amplitude for each frequency component to be plotted as a function of position in the detecting plane. Figure 2(b) shows the array direction of this phononic crystal.

To study the negative refraction imaging effect, the field patterns at the frequencies of 0.68 and $0.74 \mathrm{MHz}$ were measured in some planes parallel to the crystal surfaces $(X Y$ plane). Figures 3(a)-3(d) give the field patterns of the wave component of $0.68 \mathrm{MHz}$ at $20 a$ and the field pattern of 0.74 $\mathrm{MHz}$ at $24 a$ away from the source, without and with the sample in place, respectively. From Figs. 3(a) and 3(c), we clearly observe the circular pattern produced by the source in the absence of the phononic crystal. In the presence of the phononic crystal, the pattern became an elliptical image compressed in the $X$ direction [Figs. 3(b) and 3(d)], both at 0.68 and $0.74 \mathrm{MHz}$, attributable to the negative refractive effect. To illustrate the negative refraction effect more quan-
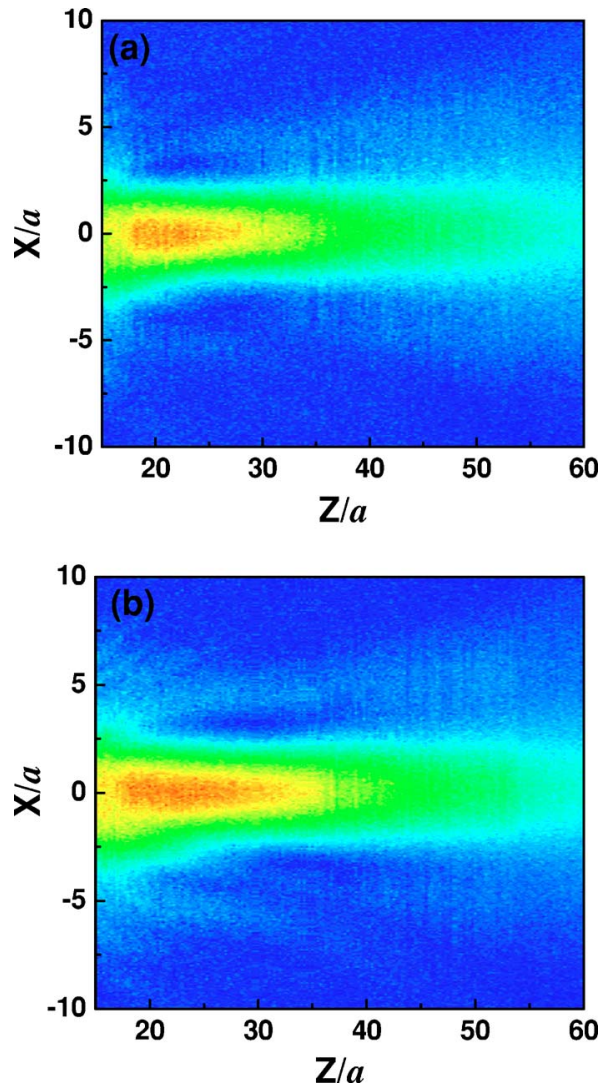

FIG. 5. (Color online) Measured field patterns in a plane parallel to the $X Z$ plane at (a) $0.68 \mathrm{MHz}$ and (b) $0.74 \mathrm{MHz}$.

titatively, we depict the amplitude of the wave field along the $X$ direction in the middle of the patterns at 0.68 and 0.74 MHz, shown in Figs. 4(a) and 4(b), respectively, where the circular symbols denote the measured field amplitudes without the phononic crystal, and the triangular symbols denote the results with the phononic crystal. The field amplitude along the $Z$ direction away from the source for 0.68 and 0.74 $\mathrm{MHz}$ are shown in Fig. 4(c). Again, it is seen that there are points of maximum intensity, signifying a focusing effect.

To further study the negative refraction imaging, we also measured the field in a plane perpendicular to the crystal surfaces and parallel to the $X Z$ plane. The experimental results at 0.68 and $0.74 \mathrm{MHz}$ are shown in Figs. 5(a) and 5(b), respectively. We can clearly see a concentration of power at

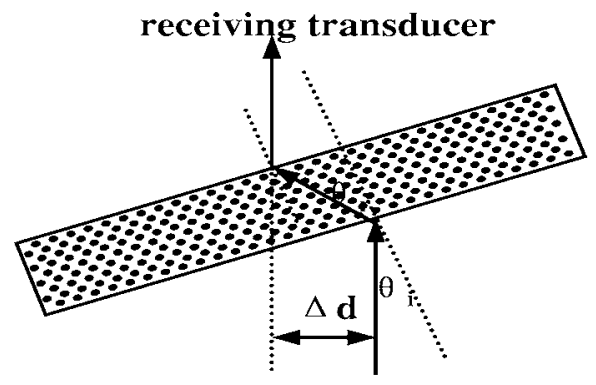

generating transducer

FIG. 6. Experimental setup for the measurement of the negative refractive index. 


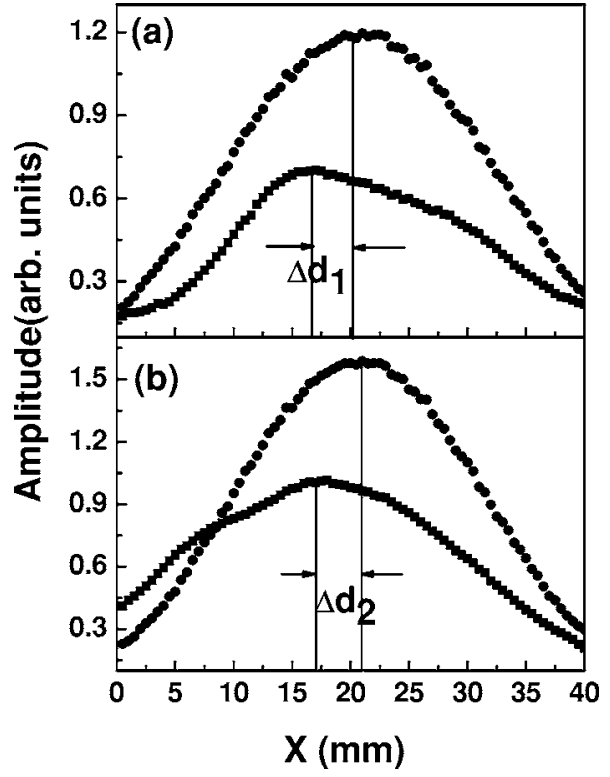

FIG. 7. Measured pressure distribution of the input (circle) and output (square) beam at (a) $0.68 \mathrm{MHz}$ and (b) $0.74 \mathrm{MHz}$.

a distance away from the sample (along the $Z$ direction) for both 0.68 and $0.74 \mathrm{MHz}$; the wave beam is about two lattice constants wide along the $X$ direction, but elongated along the $Z$ direction (about $10 a$ at $0.68 \mathrm{MHz}$ and $15 a$ at $0.74 \mathrm{MHz}$ ). Compared with the image of a point source formed by a slab of the sonic crystal having a square array, ${ }^{13}$ the image observed here has a comparatively big separation from the back surface of the sample; it is in fact a farfield image. The focused image supports the claim that a flat slab of phononic crystal can act as a lens. It is an elongated image of a point source, due to the small refractive index and the possible loss of the material. From Fig. 4(c) and Fig. 5, we can see that for different frequencies, there are different focus distances. For $0.68 \mathrm{MHz}$, the focus centers about $20 a$ from the source, but for $0.74 \mathrm{MHz}$, it is at about $24 a$.

As an extension of the above experiment, we measure the negative refraction index with another experimental scheme, shown in Fig. 6. A generating transducer was placed far away from the sample to yield an input pulse approximating a Gaussian beam. A transducer with a smaller diameter served as the receiving transducer. The incident angle is fixed at $\theta_{i}=6.42^{\circ}$; we measure the shift of the transmitted beam from the incident beam $\Delta d$ through the pressure distribution curves for a given frequency.

Figure 7 gives the measured pressure distribution curves of the input and output beams at 0.68 and $0.74 \mathrm{MHz}$, where the circle is the input beam and the square is the output beams. The Snell's law gives the refractive index $|n|$ $=\sin \theta_{i} / \sin \theta_{r}$, and from the experimental setup, the beam shift is $\Delta d=L\left(\tan \theta_{i}+\tan \theta_{r}\right) \cos \theta_{i}$, where $L$ is the thickness of the phononic crystal, $\theta_{i}$ is the incident angle, and $\theta_{r}$ is the refraction angle. From Fig. 7 , we can get $\Delta d_{1}=3.3 \mathrm{~mm}$ for

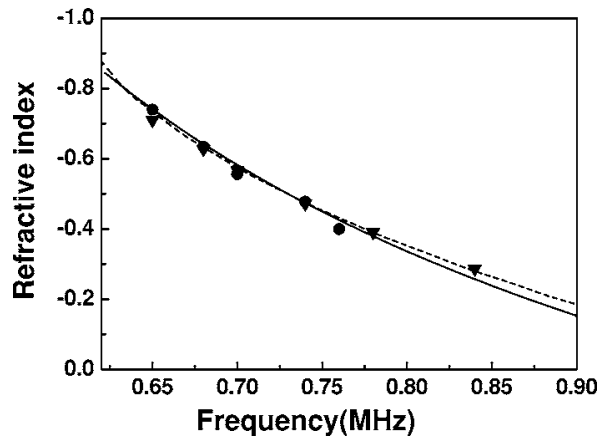

FIG. 8. The negative refraction index $n$ versus frequency. The solid line denotes theory, the dashed line denotes experimental results from the band-structure measurement, the circles are from the measurement shown in Fig. 6, and the triangles are from the measurement of $\Delta S$, the source-image distance. Please see the text for details. Excellent agreement is seen.

$0.68 \mathrm{MHz}$ and $\Delta d_{2}=4.0 \mathrm{~mm}$ for $0.74 \mathrm{MHz}$. With the measured beam shift, we obtain the negative refractive index $n$ and how its value varies with the frequency, shown in Fig. 8 as circular symbols. Also shown in Fig. 8 are the theoretical results (solid line) and the experimental results (dashed line) derived from measured band structures. According to a near-axis approximation, ${ }^{17}$ the distance from the point source to the imaging focus center $\Delta S$ can be written as $\Delta S=(1+1 /|n|) L$, which presents an additional alternative for the evaluation of the refraction index. In Fig. 8 the refractive index obtained with this method is shown as triangles. The remarkable consistency obtained from different experimental measurements serves to validate the observed negative refraction phenomenon.

\section{CONCLUDING REMARKS}

In conclusion, we have experimentally achieved acoustic negative refraction farfield imaging in the $2 \mathrm{D}$ phononic crystal and studied the negative refraction effect. The imaging is due to the negative refraction effect of the phononic crystal, which acts like a flat lens. Two separate experiments were conducted: (a) imaging of the acoustic wave by the phononic crystal and (b) the measurement of the negative refraction index of the phononic crystal. The results from both the negative refraction imaging experiment and the negative refraction index experiment are consistent with predictions from the 2D MST calculations. Negative refraction is expected to be a significant step toward novel imaging acoustic and can lead to great changes in the acoustic system design.

\section{ACKNOWLEDGMENTS}

This work is supported by the National Natural Science Foundation of China (Grants No. 50425206, No. 10174054, and No. 10418014) and the Doctoral Research Foundation of Ministry of Education of China (Grant No. 20020486013). 
*Author to whom correspondence should be addressed. Electronic address: zyliu@whu.edu.cn

${ }^{1}$ V. G. Veselago, Sov. Phys. Usp. 10, 509 (1968).

${ }^{2}$ J. B. Pendry, Phys. Rev. Lett. 85, 3966 (2000).

${ }^{3}$ M. Notomi, Phys. Rev. B 62, 10696 (2000).

${ }^{4}$ C. Luo, S. G. Johnson, J. D. Joannopoulos, and J. B. Pendry, Phys. Rev. B 65, 201104(R) (2002); Phys. Rev. B 68, 045115 (2003).

${ }^{5}$ E. Cubukcu, K. Aydin, E. Ozbay, S. Foteinopoulou, and C. M. Soukoulis, Nature 423, 604 (2003).

${ }^{6}$ D. R. Smith, W. J. Padilla, D. C. Vier, S. C. Nemat-Nasser, and S. Schultz, Phys. Rev. Lett. 84, 4184 (2000).

${ }^{7}$ R. A. Shelby, D. R. Smith, and S. Schultz, Science 292, 77 (2001).

${ }^{8}$ P. V. Parime, W. T. Lu, P. Vodo, and S. Sridhar, Nature 426, 404 (2003).

${ }^{9}$ A. Martinez, H. Miguez, A. Griol, and J. Marti, Phys. Rev. B 69, 165119 (2004).

${ }^{10}$ P. V. Parimi, W. T. Lu, P. Vodo, J. Sokoloff, J. S. Derov, and S. Sridhar, Phys. Rev. Lett. 92, 127401 (2004).

${ }^{11}$ A. Berrier, M. Mulot, M. Swillo, M. Qiu, L. Thylen, A. Talneau, and S. Anand, Phys. Rev. Lett. 93, 073902 (2004).

${ }^{12}$ X. Hu, Y. Shen, X. Liu, R. Fu, and J. Zi, Phys. Rev. E 69, 030201(R) (2004).

${ }^{13}$ X. D. Zhang and Z. Liu, Appl. Phys. Lett. 85, 341 (2004).

${ }^{14}$ M. Torres and F. R. Montero, Ultrasonics 42, 787 (2004).

${ }^{15}$ F. Cervera, L. Sanchis, J. V. Sanchez-Perez, R. Martinez-Sala, C. Rubio, F. Meseguer, C. Lopez, D. Caballero, and J. SanchezDehesa, Phys. Rev. Lett. 88, 023902 (2001).
${ }^{16}$ B. C. Gupta and Z. Ye, Phys. Rev. E 67, 036606 (2003).

${ }^{17}$ C. Qiu, X. Zhang, and Z. Liu, Phys. Rev. B 71, 054302 (2005).

${ }^{18}$ S. Yang, J. H. Page, Z. Liu, M. L. Cowan, C. T. Chan, and P. Sheng, Phys. Rev. Lett. 93, 024301 (2004).

${ }^{19}$ M. S. Kushwaha, P. Halevi, L. Dobrzynski, and B. DjafariRouhani, Phys. Rev. Lett. 71, 2022 (1993).

${ }^{20}$ M. Kafesaki, M. M. Sigalas, and N. Garcia, Phys. Rev. Lett. 85, 4044 (2000).

${ }^{21}$ Z. Y. Liu, X. X. Zhang, Y. W. Mao, Y. Y. Zhu, Z. Yang, C. T. Chan, and P. Sheng, Science 289, 1734 (2000).

${ }^{22}$ D. Garcia-Pablos, M. Sigalas, F. R. Montero de Espinosa, M. Torres, M. Kafesaki, and N. Garcia, Phys. Rev. Lett. 84, 4349 (2000).

${ }^{23}$ J. O. Vasseur, P. A. Deymier, B. Chenni, B. Djafari-Rouhani, L. Dobrzynski, and D. Prevost, Phys. Rev. Lett. 86, 3012 (2001).

${ }^{24}$ Z. Liu, C. T. Chan, P. Sheng, A. L. Goertzen, and J. H. Page, Phys. Rev. B 62, 2446 (2000).

${ }^{25}$ J. Mei, Z. Liu, J. Shi, and D. Tian, Phys. Rev. B 67, 245107 (2003).

${ }^{26}$ Y. Y. Chen and Z. Ye, Phys. Rev. E 64, 036616 (2001).

${ }^{27}$ E. Cubukcu, K. Aydin, E. Ozbay, S. Foteinopoulou, and C. M. Soukoulis, Phys. Rev. Lett. 91, 207401 (2003).

${ }^{28}$ Z. Y. Li and L. L. Lin, Phys. Rev. B 68, 245110 (2003).

${ }^{29}$ X. Zhang, Phys. Rev. B 70, 205102 (2004).

${ }^{30}$ H. Kosaka, T. Kawashima, A. Tomita, M. Notomi, T. Tamamura, T. Sato, and S. Kawakami, Phys. Rev. B 58, R10096 (1998).

${ }^{31}$ W. M. Robertson and J. F. Rudy III, J. Acoust. Soc. Am. 104, 694 (1998). 